\title{
Diferencias autonómicas en la composición de la desigualdad en la expectativa de matriculación universitaria: efectos primarios y efectos secundarios
} Regional Differences in the Composition of the Inequality in the Expectation of Enrolment in University: Primary and Secondary Effects Manuel Tomás Valdés Fernández

\section{Resumen}

El crecimiento del nivel universitario en España ha sido muy intenso durante décadas. No obstante, las desigualdades en el acceso a la universidad han permanecido. La creación del Espacio Europeo de Educación Superior trajo consigo un renovado interés por abordar la dimensión social de la universidad, especialmente en lo tocante a la equidad en el acceso al nivel universitario. A partir de la oleada 2015 del estudio PISA, el presente trabajo pone de manifiesto las muy importantes desigualdades que aún hoy perviven en la expectativa de matriculación universitaria del alumnado español de 15 años, especialmente en algunas regiones del país como la Comunidad Valenciana o las Islas Baleares. Dicha desigualdad ha sido descompuesta a partir de la noción de efectos primarios y secundarios del origen social, a fin de determinar las formas más eficaces para que las distintas regiones aborden las desigualdades en el acceso a la universidad.

\section{Palabras clave}

Efectos primarios y secundarios, desigualdad educativa, expectativa académica, dimensión social de la universidad.

\section{Abstract}

The growth of the university system in Spain has been intense during decades. Nonetheless, inequalities in the access to University have remained. The creation of the European Higher Education Area (EHEA) brought a renewed interest in addressing the social dimension of Higher Education, particularly regarding equity of access to University. Based on data from the 2015 wave of PISA, this paper shows the important inequalities that even today remain in the expectation of enrolment in University among Spanish 15 years old students, especially in certain regions such as Comunidad Valenciana or Islas Baleares. That inequality has been decomposed into primary and secondary effects of social origin, with the aim of establishing the most effective ways for the different regions to address inequality in the access to University.

\section{Keywords}

Primary and secondary effects, educational inequality, academic expectations, social dimension of University.

\section{Cómo citar/Citation}

Valdés Fernández, Manuel Tomás (2019). Diferencias autonómicas en la composición de la desigualdad en la expectativa de matriculación universitaria: efectos primarios y efectos secundarios. Revista de Sociología de la Educación-RASE, 12 (2), 209-227. http://dx.doi.org/10.7203/ RASE.12.2.14460. 


\section{La dimensión social de la universidad: luces y sombras}

El concepto de dimensión social de la Educación Superior surgió en el contexto de las discusiones para el desarrollo del Espacio Europeo de Educación Superior (EEES) que se remontan a la última década del siglo xx. No obstante, no es hasta la Conferencia de Londres, en el año 2007, cuando se pone negro sobre blanco en qué consiste esa dimensión social de la Educación Superior. La universidad no tiene solo por objetivo ofrecer trabajadores altamente formados a un mercado laboral con una creciente necesidad de cualificación, sino que debe contribuir a la cohesión social, a la reducción de las desigualdades y a la diseminación del conocimiento en la sociedad.

De esta forma, la incorporación de la dimensión social de la universidad en los debates sobre el EEES introdujo como objetivo ineludible la reducción de la desigualdad de oportunidades educativas en el acceso y finalización de estudios universitarios. Y eso no solo en defensa de un principio básico de justicia social, sino también para el mejor aprovechamiento del talento diseminado por todos los grupos sociales (Ariño Villarroya, 2014).

La preocupación por la dimensión social de la educación superior implicaba un esfuerzo por aumentar la equidad participativa en el nivel universitario, tanto en lo que hace al origen socioeconómico del alumnado, como a las posibles desigualdades por género, edad, estatus migratorio o diversidad funcional. Siguiendo a Ariño Villarroya, la equidad participativa remitiría a un ideal donde «el conjunto de estudiantes que ingresa, participa y completa los estudios universitarios, refleje la diversidad socioeconómica y cultural de nuestras sociedades» (2011: 25). Para hacerlo posible, en el mismo Comunicado de Londres (2007) se proponía mejorar el servicio educativo que se prestaba a los estudiantes en la Educación Superior, flexibilizar los itinerarios de acceso a la universidad e incrementar la participación de todos los grupos sociales en los niveles previos al universitario.

No obstante, la dimensión social de la Educación Superior y la noción de equidad participativa no se limitan al acceso a la universidad, sino que incluyen aspectos relativos a la participación activa del conjunto del alumnado en el gobierno universitario y en la vida del campus, la participación cívica y, por supuesto, la participación académica, lo que significa enfatizar no solo la igualdad de oportunidades, sino también la igualdad de resultados (Ariño Villarroya, 2011).

En el presente trabajo se abordarán esos aspectos de la equidad participativa relativos a la igualdad en el acceso a la universidad. Para ello, se estudiará la expectativa de matriculación universitaria del alumnado que hoy está matriculado en los cursos finales de la Educación Secundaria Obligatoria, analizando las posibles desigualdades que en dicho momento ya puedan existir y que, a buen seguro, condicionarán la futura composición social del alumnado universitario.

\section{El nivel universitario en España}

El renovado interés por la equidad participativa en la universidad que trajeron consigo los debates sobre el EEES vino de la mano de un hecho bien constatado: pese a un crecimiento notable de la matriculación universitaria en muchos países europeos a lo largo de la segunda mitad del siglo xx y principios del xxi, la reducción de las desigualdades en el acceso a la universidad había sido modesta.

Observando el caso español, el crecimiento cuantitativo del nivel universitario a lo largo de las últimas décadas ha sido notable en muy distintos sentidos. Atendiendo a datos ofrecidos por el Ministerio de Educación, puede comprobarse que el número de centros universitarios, el tamaño del colectivo docente, 
el número de alumnos o el presupuesto dedicado a sufragar el sistema de becas han aumentado de forma significativa, aunque no todos en la misma proporción.

De acuerdo con el Ministerio de Educación, España ha pasado de contar con 39 universidades públicas y privadas en 1990, a los 84 centros universitarios actuales. En tales centros se empleaban un total de 98710 profesores en el curso académico 2004-2005, y en poco más de una década el cuerpo docente universitario ha alcanzado los 122910 efectivos en el curso 2016-2017. El número de alumnos matriculados en la universidad española ha experimentado una evolución aún más intensa. De acuerdo con los datos ofrecidos por Ariño Villarroya (2011), en 1960 el sistema universitario español contaba con 60000 alumnos. En el año 1985 ya se habían superado los 850000 y hoy acuden a la universidad alrededor de un millón y medio de estudiantes. Y si ese alumnado disfrutó en el curso académico 2006-2007 de un sistema de becas con un presupuesto ejecutado de $641148.000 €$, diez años después se han alcanzado los $911260800 €$.

El crecimiento del nivel universitario en España es notable. Todo lo más en un contexto demográfico en el que la población de entre 18 y 24 años no ha dejado de reducirse desde mediados de los años noventa. Como resultado, la tasa neta de escolarización universitaria -proporción que la población universitaria de entre 18 y 24 años representa sobre el grupo población de 18 a 24 años- no ha hecho otra cosa que aumentar desde el año académico 2007-2008 (Gráfico 1). En el curso 2016-2017, el 32\% de los jóvenes españoles de entre 18 y 24 años se hallaba matriculado en el nivel universitario, proporción que contrasta con el 23,1\% del curso 1994-1995.

Gráfico 1. Evolución de la tasa neta de escolarización universitaria, 1994-2017

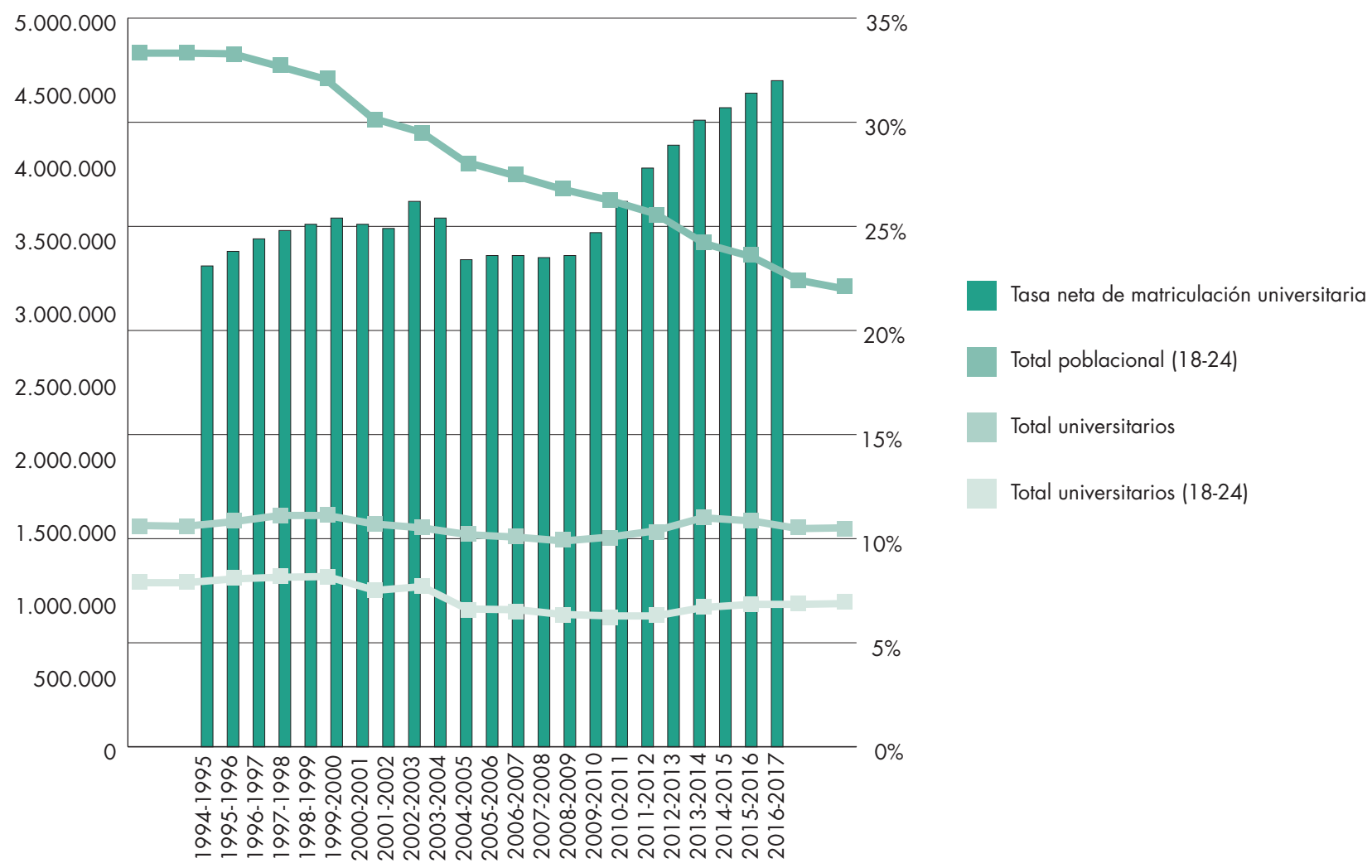


No obstante, esos números no deben dirigirnos a la autocomplacencia. Lo cierto es que ese intenso crecimiento del alumnado universitario no ha venido acompañado de una significativa reducción de la desigualdad entre clases sociales en el acceso a la universidad; al menos no una reducción equivalente al notable esfuerzo realizado en ampliar el nivel universitario. En efecto, y como apuntaran Langa Rosado y David (2006), más que a una universidad de masas, hemos derivado hacia una universidad masificada.

A lo largo de las últimas dos décadas, distintos estudios han reflejado ese estancamiento en la reducción de las desigualdades de acceso a la universidad española. Una forma habitual de valorar la desigual participación de los distintos estratos sociales en la universidad parte de la comparación del nivel educativo y estatus ocupacional de los progenitores del alumnado universitario con el de la población general o, más concretamente, con el del grupo poblacional de edad donde potencialmente se concentran los padres y madres de esos alumnos. Siguiendo dicho procedimiento, Ruesga y Heredero (2010) observaron que el nivel medio de recursos económicos anuales de los hogares de los estudiantes universitarios era un $28 \%$ superior al nivel económico medio de las familias españolas, a la vez que el nivel de estudios de los progenitores del alumnado universitario también era notablemente superior al de la población general.

Conclusiones similares se han alcanzado a partir de la explotación de la IV oleada de la encuesta Eurostudent, donde la comparación del nivel educativo y ocupación de los progenitores del alumnado universitario con la composición social del grupo poblacional de entre 40 y 60 años, permitía observar una notable sobrerrepresentación del alumnado con padres de mayor nivel educativo, mayor prestigio ocupacional y menores tasas de paro (Barañano y Finkel, 2014; Barañano, Finkel, y Rodríguez, 2011). Por ofrecer tan solo un ejemplo, el 26,9\% de los estudiantes universitarios encuestados manifestaron que su padre se desempeñaba como trabajador manual, mientras que el 47,8\% de los varones del grupo de 40-60 de la población general tenía una ocupación de tales características. Muy recientemente, Herrera (2019) ha empleado el mismo procedimiento recurriendo a datos del Ministerio de Educación y el Instituto Nacional de Estadística, mostrado no solo la persistencia de dichas desigualdades, sino su agravamiento tras la crisis económica.

El estudio del alumnado de las universidades de Barcelona y Autónoma de Barcelona llevado a cabo por Elias y Daza (2014) apuntaba en la misma dirección. Conforme a la información aportada por las autoras, la implementación del Plan Bolonia había venido acompañada de un descenso del $9 \%$ en el número de estudiantes con padres de perfiles formativos bajos. Ese descenso puede asociarse a la preferencia del alumnado de clases trabajadoras por titulaciones de menor duración y sus estrategias de compatibilización del tiempo de estudio con su participación en el mercado laboral. El estudio de Troiano y Torrents (2018) sobre el caso catalán abunda en dichas conclusiones, observando que, si bien la tasa de alumnos de nuevo ingreso con progenitores con estudios obligatorios se había mantenido constante en la segunda parte de la crisis económica, la evolución de la matrícula total en la universidad catalana de ese perfil de alumnos se redujo. Recuérdese que Cataluña fue la Comunidad Autónoma con un mayor crecimiento del precio de matrícula tras la reforma del año 2012 (Escardíbul, Pérez-Esparrells, Morales, y De la Torre, 2014).

Con anterioridad a tales estudios, la investigación de e Pablos y Gil Izquierdo (2008) ya apuntaba a principios de siglo hacia la persistencia de importantes desigualdades en el acceso a la universidad que no estaban siendo mitigadas por el crecimiento del alumnado universitario. Tras modelizar la demanda universitaria de los jóvenes españoles a partir de datos de la Encuesta de Condiciones de Vida de 2004, ambos autores evidenciaron que, dado el sistema de precios universitarios no dependiente de la renta 
existente en España, un menor poder adquisitivo del hogar ejercía un impacto muy negativo sobre la intención de acceder a la universidad.

Como se comentaba anteriormente, lo que se pretende aquí es ofrecer una aproximación a la composición social del futuro alumnado universitario a partir de la expectativa de matriculación en la universidad manifestada por los alumnos españoles que participaron en las pruebas PISA del año 2015. Para ello, nos apoyaremos en la distinción entre efectos primarios y secundarios del origen social y, aprovechando el elevado tamaño muestral a nivel regional, desagregaremos los resultados por Comunidad Autónoma. Conocer la manera en que la desigualdad en el acceso al nivel universitario se produce es fundamental para el adecuado desarrollo de políticas públicas apropiadas dadas las particularidades regionales del país.

\section{Efectos primarios y secundarios del origen social}

Las denominaciones de efectos primarios y secundarios fueron acuñadas por el sociólogo francés Raymond Boudon en el año 1974, quien pretendía describir el efecto independiente que el rendimiento académico y la toma de decisión tenían sobre el logro académico alcanzado por un alumno. Tras dos décadas sin un impacto relevante, los trabajos de Erikson y Jonsson (1996) y, especialmente, de Breen y Goldthorpe (1997), dieron nuevo lustre a la propuesta para convertirse hoy en una de las aproximaciones fundamentales al estudio de las desigualdades en la configuración del itinerario formativo de los alumnos. De la mano de la tradición iniciada por Mare $(1980,1981)$, donde los itinerarios formativos son estudiados como una sucesión de transiciones educativas, la distinción entre efectos primarios y secundarios adquirió una notable importancia tanto en el estudio de las decisiones verticales de continuación de estudios entre dos niveles del sistema de enseñanza, como en las decisiones horizontales sobre la vía curricular por la que continuar los estudios.

Boudon (1974) denominó efectos primarios a la influencia que el origen social ejerce sobre la decisión educativa de un alumno a través de su rendimiento académico. Los recursos e influencias propios de los hogares más acomodados ayudan a obtener mejores resultados en la escuela, lo que provoca una mayor ambición académica y, con ello, una mayor probabilidad de pretender completar estudios universitarios. No obstante, cuando uno compara dos alumnos de idéntico rendimiento, pero distinto origen social, observa que aún existen diferencias en la probabilidad de completar cualquier nivel del sistema de enseñanza; es decir, cuando uno controla el efecto del rendimiento académico sobre la decisión de matriculación universitaria, aún observa desigualdades entre alumnos de distinta clase social. A ello lo denominó Boudon efectos secundarios, atribuyéndolo a la forma particular en que los alumnos de diferente extracción social se enfrentan y resuelven el proceso de toma de decisiones educativas en cada nivel de rendimiento.

Para el sociólogo francés, todos los alumnos comparten una motivación idéntica por evitar la movilidad social descendente. Para lograrlo, cada alumno se pregunta qué nivel de estudios le permitiría reproducir su estatus social de origen. En tanto que los alumnos de clase baja requieren de un nivel educativo menor para mantener su estatus social, su ambición universitaria es menor (efecto suelo). En cambio, los alumnos de clases altas necesitan un título universitario si quieren ser capaces de mantener su posición en la estructura social (efecto techo). Producto del distinto riesgo de movilidad descendente al que se enfrentan los alumnos de distinta clase social, sus decisiones sobre la matriculación universitaria divergirán incluso ante situaciones de idéntico rendimiento académico.

Una forma alternativa de explicar la distinta expectativa universitaria del alumnado de diferente extracción social fue planteada por Pierre Bourdieu (1979; 2009), para quien el conjunto de experiencias 
materiales vividas por los individuos les lleva a incorporar un habitus particular, un conjunto de principios de apreciación, interpretación y percepción de la realidad que orientan la respuesta dada en una particular situación. Cuando un alumno participa en entornos sociales en los que la experiencia universitaria y el éxito académico son habituales, la percepción que desarrolla sobre la universidad es una muy particular, naturalizando la elección universitaria sin que exista una auténtica deliberación reflexiva. En cambio, cuando el alumno participa en entornos sociales pobres en tales ejemplos de éxito, la universidad se convierte en un objetivo inimaginable sin que medie ningún proceso reflexivo al respecto. Dado que los alumnos de más alta extracción social tienden a participar en entornos sociales ricos en experiencias de éxito académico y los alumnos de extracción social baja no, los primeros desarrollarán expectativas universitarias con mucha mayor facilidad para cualquier nivel de rendimiento.

Sea como fuere, la distinción entre efectos primarios y secundarios propia del estudio de transiciones educativas puede ser trasladada sin dificultades al análisis de la expectativa de matriculación universitaria. De la misma forma que la matriculación definitiva, la expectativa de matriculación estará condicionada por el origen social del alumno de dos formas. Primero, quienes pertenezcan a hogares más acomodados tendrán preferencia por completar una titulación universitaria porque habrán alcanzado mayores cotas de rendimiento y, al rendir mejor, se sentirán más capaces de responder a la exigencia académica de la universidad (efectos primarios). Segundo, quienes pertenecen a hogares más acomodados, donde la experiencia universitaria es más habitual y donde el título universitario más necesario para mantener el estatus social, desarrollarán una mayor ambición académica que quienes forman parte de hogares más humildes, donde son menos cotidianos los ejemplos de éxito universitario y menos necesaria la titulación para reproducir el nivel de vida de su origen social (efectos secundarios). Así pues, podemos descomponer la desigualdad en la expectativa de matriculación universitaria entre efectos primarios y efectos secundarios de la misma forma que se descompone la desigualdad en la matriculación definitiva en los estudios internacionales sobre transiciones educativas (Erikson y Rudolphi, 2010; Jackson, 2013; Jerrim, Chmielewski, y Parker, 2015; Neugebauer \& Schindler, 2012).

\section{Fuente de información}

El estudio de la expectativa universitaria del alumnado español se ha realizado a partir de la base de datos de la oleada 2015 de PISA, en la que participaron un total de 39066 alumnos. Además de su expectativa académica, los participantes ofrecieron amplia información sobre el estatus socioeconómico y cultural de su origen social, y completaron las pruebas de competencia matemática, lectora y científica que dan medida del desarrollo competencial alcanzado a los 15 años por el alumno. Dichas variables constituyen la información mínima necesaria para llevar a cabo la descomposición de la desigualdad observada en la expectativa universitaria entre efectos primarios y secundarios. A partir de la Comunidad Autónoma en la que el alumno cursa sus estudios de Educación Secundaria Obligatoria, se han desagregado todos los resultados a nivel regional.

Expectativa universitaria. El alumnado participante en PISA fue preguntado por el nivel educativo máximo que esperaba completar, ofreciendo las siguientes cinco alternativas de respuesta: ESO, Formación Profesional de Grado Medio, Bachillerato, Formación Profesional de Grado Superior y Universidad. Se ha construido una variable de valor 1 cuando el alumno respondió universidad y 0 en caso contrario.

Origen social. Dada la abundante información proporcionada por la base de datos PISA sobre el origen social del alumnado, se ha optado por diferenciar tres grupos sociales a partir del Índice de Estatus 
Socioeconómico y Cultural (ISEC). El ISEC se construye a partir del nivel educativo y estatus ocupacional de ambos progenitores y la posesión de bienes materiales y culturales en el hogar. El ISEC del alumno indica los recursos que las familias pueden movilizar para mejorar el rendimiento académico de sus hijos y promover el desarrollo de horizontes formativos ambiciosos. Por esa razón se considera idóneo para la descomposición que aquí se pretende entre efectos primarios y secundarios. A partir de los quintiles del ISEC, se ha distinguido un grupo de nivel socioeconómico y cultural bajo (primer quintil), uno de nivel socioeconómico y cultural alto (quinto quintil) y otro de nivel socioeconómico y cultural medio (tres quintiles intermedios).

Rendimiento. Las pruebas PISA ofrecen información sobre el desarrollo competencial del alumno en las áreas matemática, lectora y científica. Se ha calculado una puntuación de desarrollo competencial en PISA a partir de la media de rendimiento en las tres competencias anteriormente mencionadas. Debe advertirse, no obstante, que tales resultados no son perfectamente asimilables al rendimiento académico, en tanto que los alumnos de más alta extracción social tienen una mayor capacidad para convertir ese desarrollo competencial en calificaciones escolares altas (Martínez García, 2011).

Tabla I. Estadísticos descriptivos de las variables empleadas

\begin{tabular}{|c|c|c|c|c|c|}
\hline & $\mathbf{N}$ & MÍNIMO & MÁXIMO & MEDIA & DESVIACIÓN TÍPICA \\
\hline Expectativa universitaria & 38699 & $0 \%$ & $100 \%$ & $52,7 \%$ & $49,9 \%$ \\
\hline \multicolumn{6}{|l|}{ Origen social (ISEC) } \\
\hline Grupo de estatus socioeconómico y cultural bajo & 7742 & $-7,1816$ & $-1,5388$ & $-2,0966$ & 0,4532 \\
\hline Grupo de estatus socioeconómico y cultural medio & 23229 & $-1,5385$ & 0,7106 & $-0,4375$ & 0,6429 \\
\hline Grupo de estatus socioeconómico y cultural alto & 7742 & 0,7108 & 3,5163 & 1,1211 & 0,3119 \\
\hline Rendimiento en PISA & 39066 & 215,23 & 744,54 & 497,60 & 77,60 \\
\hline
\end{tabular}

Fuente: PISA 2015.

\section{Metodología del estudio}

El método más empleado para descomponer la desigualdad observada en una cierta decisión educativa entre efectos primarios y secundarios, parte de la consideración de escenarios contrafactuales en los que imaginamos qué expectativa universitaria tendría el alumnado de una clase social de haber rendido o tomado decisiones como es propio de otra clase social (Erikson, Goldthorpe, Jackson, Yaish, y Cox, 2005; Jackson, Erikson, Goldthorpe, y Yaish, 2007; Kartsonaki, Jackson, y Cox, 2013). Considerando tales escenarios junto con los escenarios factuales en que los alumnos rinden y deciden como es característico en su clase social, y partiendo de un esquema de tres clases sociales, obtenemos los nueve escenarios representados en la Tabla II. Por ejemplo, el escenario C indica la probabilidad de expectativa universitaria de un alumnado hipotético que, habiendo rendido como lo hacen los alumnos de clase baja, habría tomado decisiones como es característico entre los alumnos de clase alta.

Tabla II. Escenarios factuales y contrafactuales

\begin{tabular}{lcccc}
\hline & DECISIÓN CLASE BAJA & DECISIÓN CLASE MEDIA & DECISIÓN CLASE ALTA \\
\hline Rendimiento Clase Baja & A & B & C \\
\hline Rendimiento Clase Media & D & E & H & F \\
\hline Rendimiento Clase Alta & G & I \\
\hline
\end{tabular}


A fin de calcular las probabilidades de expectativa universitaria en los escenarios contrafactuales, Erikson et al., (2005) propusieron el siguiente modelo:

$$
P_{j k}(E U)=\int\left(\frac{1}{\sigma_{j} \sqrt{2 \pi}} e^{\frac{-\left(x-\mu_{j}\right)^{2}}{2 \sigma_{j}^{2}}}\right)\left(\frac{e^{a_{k}+b_{k} x}}{1+e^{a_{k}+b_{k} x}}\right) d x
$$

Donde el primer factor representa la distribución de rendimiento de la clase j y el segundo factor parametriza la toma de decisión para cada nivel de rendimiento de la clase k. Cuando los subíndices j y $\mathrm{k}$ hacen referencia a la misma clase social, obtendremos la probabilidad de expectativa universitaria en los escenarios factuales, y cuando hagan referencia a clases distintas, la resolución de la integral conducirá a las probabilidades en los escenarios contrafactuales.

Una vez conocidas tales probabilidades, podemos obtener una medida de efectos primarios y secundarios comparando pares de escenarios dentro de una misma fila o columna en la Tabla II. Si uno compara escenarios contenidos en una misma fila (A-B-C, D-E-F, G-H-I), observará que la distribución de rendimiento en tales escenarios permanece constante, y lo único que varía es la forma en que se toman decisiones. Es decir, toda la desigualdad que se observe entre dos escenarios de una misma fila se debe a efectos secundarios. De la misma forma, toda la desigualdad observada entre escenarios contenidos en una misma columna (A-D-G, B-E-H, C-F-I) es imputable a efectos secundarios. Sumando ambos efectos obtenemos la desigualdad total observada y calculando la proporción que cada uno representa respecto a esa desigualdad total obtenemos su importancia relativa.

Para llevar a cabo tales comparaciones, partiremos de los valores odds ratio de cada escenario, esto es, del cociente entre la probabilidad de expectativa universitaria y la probabilidad de expectativa no universitaria de cada escenario:

$$
Q_{j k}=\frac{P_{j k}}{1-P_{j k}}
$$

Tales odds ratio expresan cuánto más probable es que un alumno espere ir a la universidad a no ir bajo un escenario particular. La desigualdad total es calculada a través de un doble odds ratio o, en la terminología empleada por los proponentes del procedimiento, por un odds ratio sintético, calculado como el cociente de los odds ratio de dos escenarios factuales. Por ejemplo, la desigualdad entre las clases baja y alta se calcula dividiendo el odds ratio del escenario A entre el odds ratio del escenario I:

$$
Q_{B B \cdot A A}=\frac{Q_{B B}}{Q_{A A}}=\frac{\frac{P_{B B}}{1-P_{B B}}}{\frac{P_{A A}}{1-P_{A A}}}
$$

Cabe preguntarse en qué se distingue esta manera de expresar la desigualdad de aquella que sencillamente estudia la diferencia en la probabilidad de dos clases de mantener una expectativa universitaria (P_AA-P_BB). La manera más sencilla de explicarlo es con un ejemplo. Imaginemos una situación en la que el $30 \%$ del alumnado de clase baja y el $60 \%$ del alumnado de clase alta va a la universidad. La diferencia absoluta entre clases sería de 30 puntos porcentuales, lo que podríamos calificar como una desigualdad notable. Pensemos ahora en una segunda situación en la que el $60 \%$ del alumnado de clase baja y el 90\% del alumnado de clase alta quieren ir a la universidad. La diferencia es la misma que en el caso anterior, 
pero ¿ambos escenarios son iguales en términos de desigualdad de oportunidades? La respuesta es que no. En la primera situación, un alumno de clase alta tenía 3,5 veces más probabilidades de ir a la universidad que un alumno de clase baja (doble odds ratio $=(0,60 / 0,40) /(0,30 / 0,70))$. En el segundo escenario, en cambio, el alumno de clase alta tiene 6 veces más probabilidades de ir a la universidad que el alumno de clase baja (doble odds ratio $=(0,90 / 0,10) /(0,60 / 0,40))$. La estructura de oportunidades en que el alumno de clase alta toma su decisión de matriculación universitaria es mucho más favorable en la segunda situación que en la primera, aunque la diferencia absoluta en las tasas de matriculación entre clases sea la misma. Es obvio, por tanto, que ambas medidas reflejan dimensiones de la desigualdad diferentes y que, a efectos de la investigación que aquí nos ocupa, nos interesa analizar ambas.

De entender la forma en que la desigualdad entre clases queda recogida por los doble odds ratio, será fácil comprender el siguiente procedimiento. Obsérvese que la desigualdad entre las clases baja y alta, medida a través de su correspondiente doble odds ratio, puede descomponerse de dos formas:

$$
\begin{gathered}
Q_{B B \cdot A A}=\frac{Q_{B B}}{Q_{A A}}=\frac{\frac{P_{B B}}{1-P_{B B}}}{\frac{P_{A A}}{1-P_{A A}}}=\frac{\frac{P_{B B}}{1-P_{B B}}}{\frac{P_{B A}}{1-P_{B A}}} \times \frac{\frac{P_{B A}}{1-P_{B A}}}{\frac{P_{A A}}{1-P_{A A}}}=Q_{B B \cdot B A} \times Q_{B A \cdot A A} \\
Q_{B B \cdot A A}=\frac{Q_{B B}}{Q_{A A}}=\frac{\frac{P_{B B}}{1-P_{B B}}}{\frac{P_{A A}}{1-P_{A A}}}=\frac{\frac{P_{B B}}{1-P_{B B}}}{\frac{P_{A B}}{1-P_{A B}}} \times \frac{\frac{P_{A B}}{1-P_{A B}}}{\frac{P_{A A}}{1-P_{A A}}}=Q_{B B \cdot A B} \times Q_{A B \cdot A A}
\end{gathered}
$$

Tomando logaritmos a ambos lados en las dos expresiones, $\mathrm{L}_{\mathrm{jk}}=\ln \left(\mathrm{Q}_{\mathrm{jk}}\right)$, se convierten los anteriores productos en sumas, de forma que el efecto total del origen social sobre la decisión analizada puede ser expresado como la acción agregada de un efecto primario y un efecto secundario. Al usar logaritmos, el resultado último de desigualdad que obtengamos quedará expresado en log odds.

\section{$L_{B B \cdot A A}=L_{B A \cdot A A}+L_{B B \cdot B A}$$$
L_{B B \cdot A A}=L_{B B \cdot A B}+L_{A B \cdot A A}
$$

Calculando la proporción que cada sumando representa respecto de $\mathrm{L}_{\mathrm{BB} \cdot \mathrm{AA}}$ (desigualdad total), se obtendría la importancia relativa de cada efecto. No obstante, dado que ambas expresiones pueden conducir a resultados ligeramente diferentes, es habitual el cálculo de su media para obtener una medida definitiva de efectos primarios y efectos secundarios.

$$
\begin{aligned}
& E P(\%)=\frac{L_{B A \cdot A A}+L_{B B \cdot A B}}{L_{B B \cdot A A}} \\
& E S(\%)=\frac{L_{B B \cdot B A}+L_{A B \cdot A A}}{L_{B B \cdot A A}}
\end{aligned}
$$

Replicando tales cálculos para la desigualdad entre las clases alta y media, y las clases media y baja, obtendremos la importancia relativa en todas las comparaciones entre clases. Debe hacerse notar que, a pesar de lo habitual de dicha descomposición en el contexto internacional, su aplicación al caso español es inexistente, lo que puede explicarse fundamentalmente por la falta de datos longitudinales apropiados para el estudio de transiciones educativas en España. 


\section{Resultados}

\section{1 Desigualdades en la expectativa de matriculación universitaria por Comunidad Autónoma}

En esta primera parte de la exposición de resultados se han descrito las desigualdades absolutas en la expectativa universitaria manifestada en PISA. La Tabla III desagrega tales desigualdades en función de la Comunidad Autónoma en la que el alumno cursa sus estudios a los 15 años. Nótese, antes de continuar, que los quintiles del ISEC que han separado los tres grupos de estatus socioeconómico y cultural considerados, han sido calculados a escala nacional. Por lo tanto, el grupo de ISEC bajo de Andalucía no representa los alumnos andaluces ubicados en el primer quintil del ISEC de la muestra de Andalucía, sino los alumnos ubicados en el primer quintil del ISEC nacional que cursan sus estudios en la comunidad andaluza.

Aclarado este extremo, podemos comentar los resultados mostrados en la Tabla III. Obsérvese, en primer lugar, que el 52,8\% de los alumnos españoles participantes en PISA manifestó su intención de completar un grado universitario. Como podía anticiparse, ese porcentaje supera holgadamente la proporción de alumnos que finalmente accederán a la universidad. Recuérdese que, como vimos en el epígrafe introductorio de este artículo, la tasa neta de matriculación universitaria en España fue, en el curso 2016-2017, de 32,1\%, veinte puntos porcentuales por debajo del porcentaje de alumnos que hoy manifiestan su intención de llegar a la universidad.

Siguiendo en el nivel nacional, observamos una diferencia muy notable en la expectativa universitaria de los grupos de estatus socioeconómico y cultural construidos: mientras que el 78,8\% de los alumnos de ISEC alto pretende acceder a la universidad, tan solo el 28,8\% del alumnado de ISEC bajo espera lo propio. Una desigualdad de 50 puntos porcentuales que se trasladará en buena medida y con el paso de los años a la matriculación definitiva. Es muy probable, no obstante, que sea el alumnado de mayor estatus socioeconómico y cultural quien sea hoy excesivamente optimista respecto a sus posibilidades de acceder a la universidad, de manera que las desigualdades aquí observadas tenderán a reducirse tras el paso por la Educación Secundaria Superior.

En cuanto al grupo de ISEC medio, su expectativa universitaria fue 13 puntos superior a la del grupo de ISEC bajo y 27 puntos inferior a la del grupo de ISEC alto. Tomando las tres comparaciones en conjunto, podemos afirmar que, aunque hay diferencias significativas entre los grupos de ISEC bajo y medio, es la pertenencia al grupo de ISEC alto la que dispara la expectativa de matriculación universitaria.

Si desagregamos los datos por Comunidad Autónoma, observamos que las regiones con una expectativa universitaria más elevada son Cataluña y la Comunidad de Madrid, donde el 59,4\% y el 59,1\% del alumnado de 15 años espera acceder a la universidad, respectivamente. En el polo opuesto se sitúan Canarias y Andalucía, donde el $45 \%$ y el 56,9\% del alumnado manifestó su intención de matricularse en el nivel universitario. El resto de las regiones se sitúan dentro de los 14,4 puntos porcentuales de diferencia que separan el alumnado catalán del canario.

Considerando ahora la expectativa universitaria de los distintos grupos de estatus socioeconómico y cultural por Comunidad Autónoma, la región donde el grupo de ISEC bajo espera ir a la universidad en mayor proporción es Castilla y León, con un 34,3 \%, mientras que la que presenta la menor expectativa universitaria en el grupo de ISEC bajo es La Rioja, con un 21,1\%. Parece que la pertenencia al 
grupo de ISEC bajo es especialmente desmotivadora para el alumnado riojano. Del lado contrario, la comunidad donde el alumnado de ISEC alto presenta una mayor expectativa universitaria es Baleares, con un $84,7 \%$, mientras que la expectativa más baja entre alumnos de ISEC alto es la de País Vasco, con un $73,7 \%$. Los alumnos vascos son quienes sacan menos partido en términos de ambición académica del hecho de pertenecer al grupo de ISEC alto.

En términos de desigualdad absoluta, la región donde la diferencia en la expectativa universitaria entre los grupos de ISEC bajo y alto es mayor es la Comunidad Valencia, con una distancia de 56,3 puntos porcentuales, seguida de La Rioja con 54,6 puntos. La diferencia más baja la encontramos en Castilla y León, donde 40,9 puntos porcentuales separan la expectativa universitaria de los grupos alto y bajo, seguida de País Vasco con 43,3 puntos de diferencia. Es decir, encontramos significativas desigualdades en la expectativa universitaria a nivel nacional que, no obstante, muestran una importante disparidad regional que pasamos a analizar ahora en mayor profundidad.

Tabla III. Expectativa de matriculación universitaria por Comunidad Autónoma y origen social

\begin{tabular}{|c|c|c|c|c|}
\hline COMUNIDAD AUTÓNOMA & ISEC BAJO & ISEC MEDIO & ISEC ALTO & TOTAL \\
\hline Andalucía & $26,3 \%$ & $50,7 \%$ & $79,3 \%$ & $46,9 \%$ \\
\hline Aragón & $29,4 \%$ & $50,7 \%$ & $80,5 \%$ & $54,0 \%$ \\
\hline Asturias & $28,3 \%$ & $50,1 \%$ & $79,7 \%$ & $53,2 \%$ \\
\hline Islas Baleares & $31,5 \%$ & $54,8 \%$ & $84,7 \%$ & $54,3 \%$ \\
\hline Islas Canarias & $26,5 \%$ & $46,2 \%$ & $79,7 \%$ & $45,0 \%$ \\
\hline Cantabria & $23,6 \%$ & $49,0 \%$ & $76,4 \%$ & $50,7 \%$ \\
\hline Castilla y León & $34,3 \%$ & $53,7 \%$ & $75,2 \%$ & $54,2 \%$ \\
\hline Castilla - La Mancha & $30,4 \%$ & $48,4 \%$ & $79,4 \%$ & $49,4 \%$ \\
\hline Cataluña & $32,6 \%$ & $57,2 \%$ & $82,4 \%$ & $59,4 \%$ \\
\hline Extremadura & $31,6 \%$ & $52,8 \%$ & $78,7 \%$ & $50,0 \%$ \\
\hline Galicia & $29,0 \%$ & $53,2 \%$ & $78,7 \%$ & $53,1 \%$ \\
\hline La Rioja & $21,1 \%$ & $52,2 \%$ & $75,7 \%$ & $50,4 \%$ \\
\hline Madrid & $30,0 \%$ & $52,6 \%$ & $79,5 \%$ & $59,1 \%$ \\
\hline Murcia & $31,8 \%$ & $53,5 \%$ & $80,0 \%$ & $51,4 \%$ \\
\hline Navarra & $27,3 \%$ & $46,2 \%$ & $76,1 \%$ & $49,9 \%$ \\
\hline País Vasco & $30,4 \%$ & $53,9 \%$ & $73,7 \%$ & $55,9 \%$ \\
\hline Comunidad Valenciana & $26,6 \%$ & $54,1 \%$ & $82,9 \%$ & $54,5 \%$ \\
\hline España & $28,8 \%$ & $52,1 \%$ & $78,8 \%$ & $52,8 \%$ \\
\hline
\end{tabular}

Fuente: elaboración propia a partir de PISA 2015.

\subsection{Diferencias en la estructura de oportunidades universitarias por Comunidad Autónoma}

Una vez descrita la desigualdad en la expectativa de matriculación universitaria en términos absolutos, analicemos ahora la estructura de oportunidades en que los alumnos de las distintas Comunidades Autónomas toman sus decisiones educativas. Para ello se han calculado los doble odds ratio que comparan los grupos de estatus socioeconómico y cultural alto, medio y bajo. La Tabla IV incluye además 
la desigualdad entre grupos expresada en log odds (logaritmo natural del doble odds ratio), que será la medida de desigualdad que se descompondrá entre efectos primarios y secundarios del origen social en el siguiente epígrafe.

Ya se explicó en el apartado metodológico en qué difería una definición de desigualdad en términos absolutos de otra que remita a la estructura de oportunidades en que los alumnos de distinta extracción social toman sus decisiones educativas. Podemos ejemplificar esa distinción con los datos de que disponemos ahora observando las comunidades de Baleares y Canarias. Como pudo comprobarse en la anterior Tabla III, ambas regiones presentan una desigualdad en la expectativa universitaria entre los alumnos de ISEC alto y bajo muy similar (53,1\% y 53,2\%, respectivamente). En cambio, difieren de forma significativa en el doble odds ratio que compara ambos grupos en la Tabla IV: mientras que en Baleares es 12 veces más probable que un alumno de ISEC alto espere ir a la universidad que un alumno de ISEC bajo, en Canarias es tan solo 10 veces más probable. Es decir, que, aunque la distancia absoluta que separa a ambos grupos sociales es la misma, la estructura de oportunidades en la que se toman las decisiones educativas es más favorable al alumnado de ISEC alto en Baleares que en Canarias.

Aclarados de nuevo la forma y el sentido de la interpretación de la desigualdad que se refleja en la Tabla IV, podemos afirmar que, a nivel nacional, es 9 veces más probable que un alumno de ISEC alto espere ir a la universidad que un alumno de ISEC bajo. Esa desigualdad, a todas luces notable, diverge de forma significativa entre Comunidades Autónomas. La Comunidad Valenciana es, de nuevo, la región que presenta una estructura de oportunidades más desfavorable para el alumnado de ISEC bajo, donde es 13 veces menos probable que ese alumnado espere matricularse en la universidad que el alumnado de ISEC alto.

El dato de la Comunidad Valenciana es muy superior al resto de regiones de España, especialmente a la comunidad castellanoleonesa, donde es «solo» 5,75 veces más probable que un alumno de ISEC alto mantenga una expectativa universitaria que un alumno de ISEC bajo. Eso no quiere decir que los alumnos de Castilla y León tomen sus decisiones en un contexto igualitario, pero es obvio que la estructura de oportunidades percibida por ese alumnado cuando tome la decisión de matriculación universitaria será muy diferente a la que experimentan los alumnos valencianos. Para poner en valor el dato de Castilla y León, nótese que la desigualdad que separa al alumnado de ISEC alto y bajo en dicha región es similar a la que separa la expectativa universitaria de los alumnos de ISEC medio y bajo en Baleares (doble odds ratio de 4,67).

Tabla IV. Desigualdad de oportunidades educativas en la expectativa universitaria por Comunidad Autónoma

\begin{tabular}{lcccccc}
\hline \multirow{2}{*}{ COMUNIDAD AUTÓNOMA } & \multicolumn{2}{c}{ ISEC ALTO/ISEC BAJO } & \multicolumn{2}{c}{ ISEC MEDIO/ISEC BAJO } & \multicolumn{2}{c}{ ISEC ALTO/ISEC MEDIO } \\
\cline { 2 - 8 } & $\begin{array}{c}\text { DOBLE ODDS } \\
\text { RATIO }\end{array}$ & $\begin{array}{c}\text { DESIGUALDAD } \\
\text { EN LOG ODDS }\end{array}$ & $\begin{array}{c}\text { DOBLE ODDS } \\
\text { RATIO }\end{array}$ & $\begin{array}{c}\text { DESIGUALDAD } \\
\text { EN LOG ODDS }\end{array}$ & $\begin{array}{c}\text { DOBLE ODDS } \\
\text { RATIO }\end{array}$ & $\begin{array}{c}\text { DESIGUALDAD } \\
\text { EN LOG ODDS }\end{array}$ \\
\hline Andalućía & 10,78 & 2,38 & 3,72 & 1,31 & 2,90 & 1,06 \\
\hline Aragón & 9,95 & 2,30 & 4,08 & 1,41 & 2,44 & 0,89 \\
\hline Asturias & 10,10 & 2,31 & 3,98 & 1,38 & 2,54 & 0,93 \\
\hline Islas Baleares & 12,26 & 2,51 & 4,67 & 1,54 & 2,63 & 0,97 \\
\hline Islas Canarias & 10,17 & 2,32 & 4,35 & 1,47 & 2,34 & 0,85 \\
\hline Cantabria & 10,43 & 2,34 & 3,44 & 1,23 & 3,04 & 1,11 \\
\hline
\end{tabular}


Diferencias autonómicas en la composición de la desigualdad en la expectativa de matriculación universitaria: efectos primarios y efectos secundarios

\begin{tabular}{lcccccc}
\hline \multirow{2}{*}{ COMUNIDAD AUTÓNOMA } & \multicolumn{2}{c}{ ISEC ALTO/ISEC BAJO } & \multicolumn{2}{c}{ ISEC MEDIO/ISEC BAJO } & \multicolumn{2}{c}{ ISEC ALTO/ISEC MEDIO } \\
\cline { 2 - 7 } & $\begin{array}{c}\text { DOBLE ODDS } \\
\text { RATIO }\end{array}$ & $\begin{array}{c}\text { DESIGUALDAD } \\
\text { EN LOG ODDS }\end{array}$ & $\begin{array}{c}\text { DOBLE ODDS } \\
\text { RATIO }\end{array}$ & $\begin{array}{c}\text { DESIGUALDAD } \\
\text { EN LOG ODDS }\end{array}$ & $\begin{array}{c}\text { DOBLE ODDS } \\
\text { RATIO }\end{array}$ & $\begin{array}{c}\text { DESIGUALDAD } \\
\text { EN LOG ODDS }\end{array}$ \\
\hline Castilla y León & 5,75 & 1,75 & 2,62 & 0,96 & 2,20 & 0,79 \\
\hline Castilla - La Mancha & 8,88 & 2,18 & 4,15 & 1,42 & 2,14 & 0,76 \\
\hline Cataluña & 9,73 & 2,27 & 3,57 & 1,27 & 2,73 & 1,00 \\
\hline Extremadura & 8,05 & 2,09 & 3,33 & 1,20 & 2,42 & 0,88 \\
\hline Galicia & 9,17 & 2,22 & 3,33 & 1,20 & 2,75 & 1,01 \\
\hline La Rioja & 11,73 & 2,46 & 2,84 & 1,04 & 4,13 & 1,42 \\
\hline Madrid & 9,14 & 2,21 & 3,57 & 1,27 & 2,56 & 0,94 \\
\hline Murcia & 8,46 & 2,14 & 3,49 & 1,25 & 2,43 & 0,89 \\
\hline Navarra & 8,33 & 2,12 & 3,70 & 1,31 & 2,25 & 0,81 \\
\hline País Vasco & 6,44 & 1,86 & 2,39 & 0,87 & 2,69 & 0,99 \\
\hline Comunidad Valenciann & 13,40 & 2,60 & 4,17 & 1,43 & 3,22 & 1,17 \\
\hline España & 9,19 & 2,22 & 3,45 & 1,24 & 2,67 & 0,98
\end{tabular}

Fuente: elaboración propia a partir de PISA 2015.

\subsection{Importancia relativa de los efectos primarios y secundarios del origen social en la expectativa de matriculación universitaria por Comunidad Autónoma}

Una vez conocida la desigualdad en la expectativa universitaria expresada en log odds (Tabla IV), podemos calcular qué porcentaje de esa desigualdad es imputable al efecto del origen social sobre el rendimiento (efectos primarios) y qué porcentaje es imputable al efecto del origen social sobre la toma de decisión una vez controlado el rendimiento (efectos secundarios). Para ello se han aplicado los cálculos descritos en el apartado metodológico y los resultados quedan recogidos en los Gráficos 2, 3 y 4.

A nivel nacional, recordemos, 50 puntos porcentuales separaban la expectativa universitaria del alumnado de ISEC alto y bajo, y era 9 veces más probable que los primeros desarrollasen esa expectativa universitaria que los segundos. Pues esa desigualdad es producida en un 48,9\% a través del efecto que el origen ejerce sobre el rendimiento del alumno y en un 51,1\% a través de la forma en que se resuelve el proceso de toma de decisión tras alcanzar un cierto rendimiento previo. El porcentaje de efectos secundarios se reduce ligeramente en la comparación nacional entre alumnos de ISEC bajo y medio, y aumenta algo al comparar alumnos de ISEC alto y medio. Expresado de forma más clara, alrededor de la mitad de la desigualdad observada en la expectativa de matriculación universitaria de los alumnos españoles a los 15 años se genera independientemente del rendimiento académico alcanzado.

Tales resultados ofrecen ya información de gran valor para el desarrollo de estrategias con las que reducir la desigualdad educativa y trabajar en favor de la equidad participativa en la universidad. Pero lo que nos muestran los Gráficos 2, 3 y 4 es una significativa variabilidad en la composición de la desigualdad a nivel regional.

El Gráfico 2 descompone la desigualdad observada entre los alumnos de ISEC alto y bajo. De un lado, encontramos un grupo de regiones donde la mayor parte de la desigualdad se genera a través de efectos secundarios, como son Galicia (61,7\%), la Comunidad Valencia (57,7\%) o Madrid (56,1\%); es decir, comunidades donde la desigualdad observada se debe principalmente a la forma en que los alumnos de distinta extracción social resuelven el proceso de toma de decisiones educativas. Del otro 
lado, en comunidades como Extremadura (56,6\%), Navarra (54,7\%) o Canarias (53,5\%), los efectos primarios son mayoritarios, haciendo que la desigualdad se genere en mayor medida a través del efecto del origen social sobre el rendimiento de los alumnos. Parece obvio que, desde un punto de vista de política pública, no puede tratarse igual la desigualdad observada en Galicia que la que encontramos en Extremadura.

La comparación entre los grupos de ISEC medio y bajo representada en el Gráfico 3 ofrece una mayor variabilidad entre Comunidades Autónomas. Por un lado, comunidades como Galicia o la Comunidad Valenciana presentan unos efectos secundarios de alrededor de 60 puntos porcentuales. Por otro lado, encontramos regiones como Navarra, Murcia, Asturias o Aragón, donde los efectos primarios dan cuenta de en torno a dos tercios de la desigualdad total.

Por último, el Gráfico 4 presenta la descomposición de la desigualdad en la comparación de los grupos de ISEC alto y medio. Las comunidades aragonesa (61,9\%), gallega (59,7\%) y valenciana $(58,8 \%)$ son las tres con mayores efectos secundarios. En cambio, no se encuentra ninguna región con significativos efectos primarios, siendo Cantabria, con un 52,5\%, la que presenta unos efectos primarios más relevantes.

Solo recalcar al respecto de tales resultados la importante disparidad regional, con comunidades como Galicia o la Comunidad Valenciana donde sistemáticamente aparecen notables efectos secundarios, y regiones como Navarra donde los efectos primarios parecen ser claramente preponderantes. Es necesario ser conscientes, por tanto, de la forma en que la desigualdad educativa es generada a nivel regional. Sería absurdo concentrar los recursos e instrumentos dirigidos a abordar la desigualdad en el acceso a la universidad en una serie de programas dirigidos a atender situaciones de bajo desempeño escolar en aquellas regiones en las que la mayor parte de esas desigualdades permanecen una vez controlado el rendimiento académico, esto es, en regiones con unos elevados efectos secundarios.

\section{Gráfico 2. Composición de la desigualdad en la expectativa universitaria entre el grupo de ISEC alto y bajo}

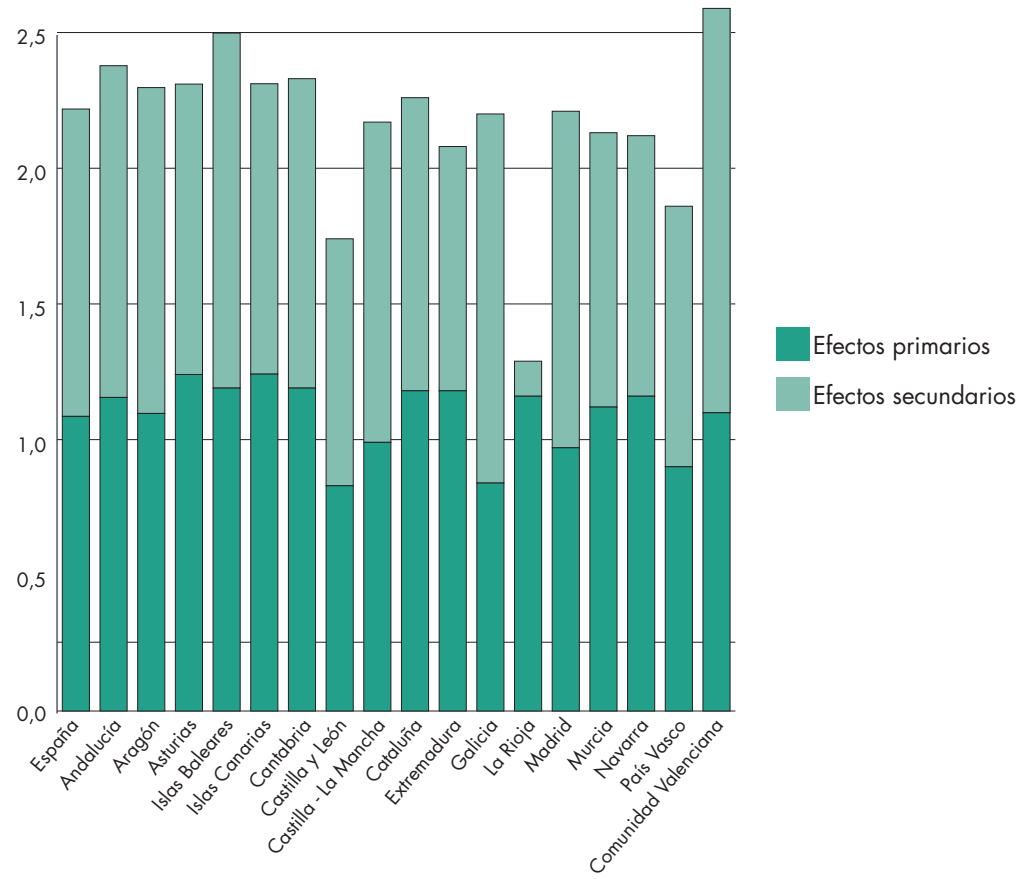

Fuente: elaboración propia a partir de PISA 2015. 


\section{Gráfico 3. Composición de la desigualdad en la expectativa universitaria entre el grupo de ISEC medio y bajo}

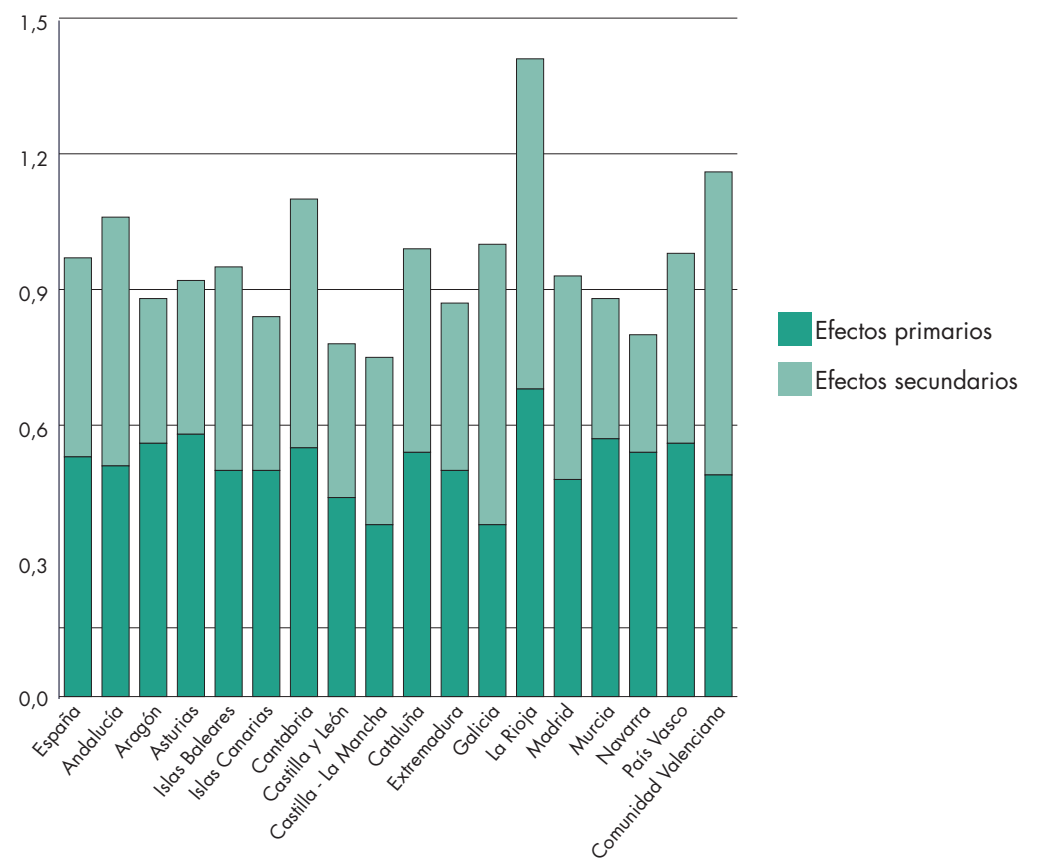

Fuente: elaboración propia a partir de PISA 2015.

\section{Gráfico 4. Composición de la desigualdad en la expectativa universitaria entre el grupo de ISEC alto y medio}

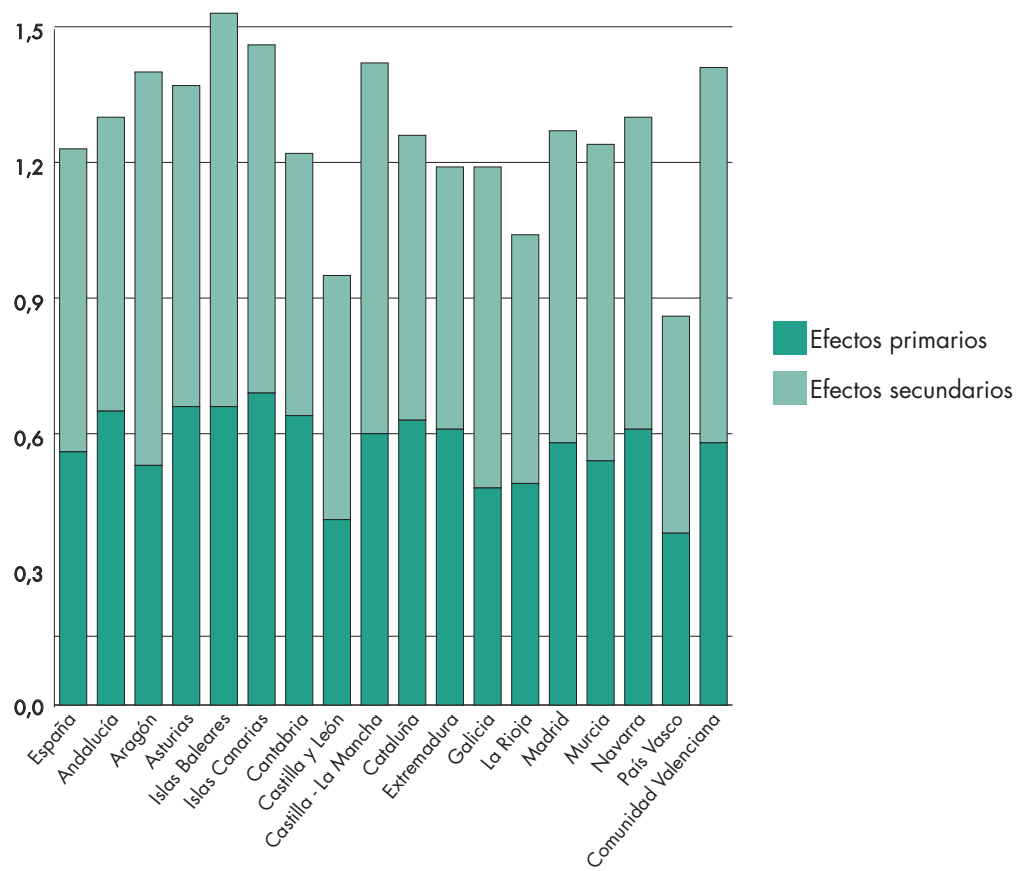

Fuente: elaboración propia a partir de PISA 2015.

\section{Conclusiones}

Para algunos investigadores, la igualdad de oportunidades remite a una situación en que no existen barreras económicas o normativas que impidan a ciertos alumnos el acceso a los distintos niveles del sistema de enseñanza. A partir del momento en que un sistema de becas y precios universitarios posibilite que 
todo aquel que desee estudiar en la universidad pueda hacerlo, las decisiones que después tomen los alumnos constituyen un ejercicio de su libertad para elegir su futuro formativo y profesional en el que la política pública poco puede y poco debe hacer. Si el alumnado de más baja extracción social tiende a elegir en menor medida la universidad, tendrá razones fundadas para hacerlo, puesto que si quisiera matricularse en el nivel universitario, no encontraría barreras normativas o económicas que se lo impidiesen (Carabaña y Rivière, 2017). No obstante, cincuenta puntos porcentuales de diferencia en la expectativa de matriculación universitaria de los grupos de ISEC alto y baja hacen difícil sostener que el acceso a la universidad en España se produce en condiciones de igualdad.

Debe enfatizarse, eso sí, que lo que se ha expuesto aquí son desigualdades en la expectativa de matriculación universitaria, no en la matriculación definitiva. Como se avanzó algo más arriba, sabemos que no todos los alumnos que desean ir a la universidad a los 15 años finalmente lo logran. Y dada la prevalencia de la opción universitaria entre el alumnado de mayor ISEC (78,8\%), parece fácil pensar que serán estos alumnos quienes verán frustrada su expectativa en mayor proporción. Su optimismo deberá enfrentarse a la realidad de la educación postobligatoria, donde no siempre serán capaces de responder a la exigencia planteada por mucho que su entorno haya construido para ellos una expectativa formativa que gravita alrededor de la universidad. Como resultado, es de esperar cierta rebaja de la desigualdad al pasar de la expectativa de matriculación a la matriculación definitiva en el nivel universitario. Ahora bien, podemos afirmar con rotundidad que las desigualdades en la expectativa universitaria aquí descritas no van a desaparecer tras el paso por la Educación Secundaria Superior, y que sesgarán notablemente la composición del alumnado universitario de la próxima década.

Más allá de esas desigualdades en términos absolutos, se ha tratado de enfatizar las importantes diferencias en la estructura de oportunidades en que los alumnos de distinto estatus socioeconómico y cultural toman sus decisiones educativas. La sociología bourdiana enfatizó la importancia de las experiencias cotidianas y las condiciones materiales de existencia en la configuración de la percepción de la realidad (Bourdieu, 1979). Es así como ciertos alumnos naturalizan la decisión de matriculación en la universidad, producto de la frecuencia con que la experiencia universitaria y el éxito educativo son vividos en los entornos sociales en los que esos alumnos participan. Y es así también como otros alumnos jamás se plantean matricularse en el nivel universitario, pues sus relaciones cotidianas no les acercan a casos de éxito académico que hayan pasado por la universidad. La construcción de lo posible y lo imposible se realiza de acuerdo a experiencias cotidianas, a entendimientos compartidos con alumnos que son identificados como iguales a uno mismo. Es así como la estructura percibida de oportunidades educativas se vuelve crucial en la toma de decisión de los alumnos de distinta extracción social. La ventaja del alumnado de ISEC alto respecto del alumnado de ISEC bajo en la Comunidad Valenciana es mayor que la que caracteriza a Castilla y León porque la probabilidad de que un alumno de ISEC alto naturalice la decisión de ir a la universidad es mucho mayor, producto de una estructura de oportunidades mucho más favorable.

Si el sistema educativo no es capaz, en sus etapas obligatorias, de impulsar la ambición formativa del alumno de menor estatus socioeconómico y cultural, que existan o no barreras económicas es irrelevante, pues quienes realmente podrían encontrárselas no llegan a plantearse jamás la opción de acceder a la universidad. Y para corregir esa desigualdad, uno debe tener muy presente la manera en que ésta se genera.

Hemos podido comprobar cómo, a nivel nacional, más de la mitad de la desigualdad observada en la expectativa de matriculación universitaria a los 15 años es producida de forma independiente al rendi- 
miento alcanzado por el alumno. Por tanto, pensar en corregir dicha desigualdad exclusivamente mejorando el desempeño escolar del alumnado en las etapas obligatorias dejaría inalterada el 51,1\% de la desigualdad total. Y, pese a ello, es precisamente así como hemos tratado de abordar la desigualdad educativa en las últimas décadas, desarrollando programas dirigidos a atender situaciones de bajo rendimiento durante la Educación Secundaria Obligatoria (educación compensatoria, desdobles por nivel de aprendizaje, las condiciones para la repetición de curso, el Programa de Refuerzo, Orientación y Apoyo, la Formación Profesional Básica, los Programas de Mejora del Aprendizaje y el Rendimiento,...).

Además, se ha mostrado una notable variabilidad regional en la forma en que la desigualdad en la expectativa universitaria es producida a nivel autonómico. Promover la equidad participativa en el nivel universitario exige reconocer y tener en cuenta las importantes diferencias regionales en el volumen y composición de la desigualdad observada en la expectativa universitaria. No es igual abordar la desigualdad castellanoleonesa que la valenciana, porque la segunda es notablemente superior a la primera. Y tampoco es lo mismo abordar la desigualdad gallega o la extremeña, porque en la primera las desigualdades se deben fundamentalmente a la forma en que los alumnos deciden una vez alcanzan un cierto nivel de rendimiento (efectos secundarios), y en la segunda se deben al efecto del origen social sobre el rendimiento académico previo (efectos primarios). Ignorar dicha heterogeneidad significa recetar los mismos programas contra la desigualdad a regiones que tienen problemas diferentes.

En conclusión, trabajar en la dimensión social de la Educación Superior y, particularmente, en la equidad participativa en la universidad, obliga a dirigir los esfuerzos mucho antes del nivel universitario. Es obvio que un sistema de becas universitarias adecuadamente dotado y configurado es fundamental para eliminar las barreras económicas a las que se enfrenta el alumnado de origen social más humilde, pero no es menos cierto que ya en la Educación Secundaria Obligatoria existen desigualdades más que notables en el horizonte formativo que los alumnos de distinta extracción social definen para sí mismos. Sin programas que estimulen y promuevan la ambición académica del alumnado de más baja extracción social, será imposible acercarnos a ese ideal de equidad participativa en el que la composición social del alumnado universitario reproduce aquella de la población general.

\section{Referencias bibliográficas}

Ariño Villarroya, Antonio (2011): "La dimensión social y la participación universitaria” en Antonio Ariño Villarroya y Ramón Llopis (eds.). ¿Universidad sin clases? Condiciones de vida de los estudiantes universitarios en España (Eurostudent IV). Madrid: Subdirección General de Documentación y Publicaciones.

Ariño Villarroya, Antonio (2014): “La dimensión social en la educación superior". Revista de Sociología de la Educación, 7 (1), 17-41.

Barañano, Margarita y Finkel, Lucila (2014): “Transmisión intergeneracional y composición social de la población estudiantil universitaria española: cambios y continuidades”. Revista de Sociología de la Educación, 7 (1), 42-60.

Barañano, Margarita, Finkel, Lucila y Rodríguez, Elena (2011). "Procedencia sociofamiliar" en Antonio Ariño Villarroya y Ramón Llopis (eds.). ¿Universidad sin clases? Condiciones de vida de los estudiantes universitarios en España (Eurostudent IV). Madrid: Subdirección General de Documentación y Publicaciones.

Boudon, Raymond (1974). Education, Opportunity, and Social inequality: Changing prospects in Western society. New York: Wiley. 
Bourdieu, Pierre (1979). La distinción. Criterio y bases sociales del gusto. Madrid: Taurus.

Bourdieu, Pierre y Passeron, Jean-Claude (2009). Los herederos: Los estudiantes y la cultura. Buenos Aires: Siglo XXI Editores Argentina.

Breen,RichardyGoldthorpe,JohnH.(1997): "ExplainingEducationDifferentials:TowardsaFormalRational Action Theory”. Rationality and Society, 9 (3), 275-305. https://doi.org/10.1177/104346397009003002.

Carabaña, Julio y Rivière, Jaime (2017): “¿La igualdad de oportunidades era esto? Estratificación, educación, desigualdad y pobreza. Conversación con Julio Carabaña". Encrucijadas - Revista Crítica de Ciencias Sociales, 14, 1-20.

Elias Andreu, Marina y Daza Pérez, Lidia (2014): "Sistema de becas y equidad participativa en la universidad". Revista de Sociología de la Educación, 7 (1), 233-251.

Erikson, Robert; Goldthorpe, John H.; Jackson, Michelle; Yaish, Meir y Cox, D. R. (2005): “On class differentials in educational attainment". Proceedings of the National Academy of Sciences, 102 (27), 97309733. https://doi.org/10.1073/pnas.0502433102.

Erikson, Robert y Jonsson, Jan O. (1996). Can Education be Equalized? The Swedish Case in Comparative Perspective. Boulder: Westview Press.

Erikson, Robert y Rudolphi, Frida (2010). "Change in Social Selection to Upper Secondary School Primary and Secondary Effects in Sweden”. European Sociological Review, 26 (3), 291-305. https://doi. org/10.1093/esr/jcp022.

Escardíbul, Josep O.; Pérez Esparrells; Carmen, Morales; Ssusana y de la Torre, Eva (2014): "Precios de matrícula en las enseñanzas universitarias por Comunidades Autónomas (1992-2013): un análisis antes y después del Espacio Europeo de Educación Superior”. XXI Encuentro de Economía Pública, 1-27.

Herrera Cuesta, Damián (2019): “¿Quién estudia en la universidad? La dimensión social de la universidad española en la segunda década del siglo XXI”. Revista de Sociología de la Educación, 12 (1), 7-23. https:/ / doi.org/10.7203/RASE.12.1.13117.

Jackson, Michelle (2013). Determined to succeed? Performance vs choice in educational attainment. California: Stanford University Press.

Jackson, Michelle; Erikson, Robert; Goldthorpe, John H. y Yaish, Mier (2007): "Primary and Secondary Effects in Class Differentials in Educational Attainment: The Transition to A-Level Courses in England and Wales”. Acta Sociologica, 50 (3), 211-229. https://doi.org/10.1177/0001699307080926.

Jerrim, John; Chmielewski, Anna K. y Parker, Phil (2015): "Socioeconomic Inequality in Access to HighStatus Colleges: A Cross-Country Comparison". Research in Social Stratification and Mobility, 42, 20-32. https://doi.org/10.1016/j.rssm.2015.06.003.

Kartsonaki, Christiana; Jackson, Michell y Cox, David R. (2013): "Primary and secondary effects. Some methodological issues" en Michelle Jackson (ed). Determined to succeed? Performance vs choice in educational attainment. California: Stanford University Press.

Langa Rosado, Delia y David, Miriam (2006): “A Massive University or a University for the Masses? Continuity and Change in Higher Education in Spain and England". Journal of Education Policy, 21 (3), 343-365. https://doi.org/10.1080/02680930600600630. 
Mare, Robert D. (1980): "Social Background and School Continuation Decisions". Journal of the American Statistical Association, 75 (370), 295-305. https://doi.org/10.2307/2287448.

Mare, Robert D. (1981): “Change and stability in educational stratification”. American Sociological Review, $46(1), 72-87$.

Martínez García, José Saturnino (2011): "Género y origen social: diferencias grandes en fracaso escolar administrativo y bajas en rendimiento educativo”. Revista de Sociología de la Educación, 4 (3), 270-285.

Neugebauer, M. y Schindler, S. (2012): “Early Transitions and Tertiary Enrolment: The Cumulative Impact of Primary and Secondary Effects on Entering University in Germany". Acta Sociologica, 55 (1), 19-36. https://doi.org/10.1177/0001699311427747.

Ruesga Benito, Santos S. y Heredero de Pablos, María I. (2010). Igualdad en el acceso y la estancia en la universidad en España. Madrid: Ministerio de Educación y Ciencia.

Troiano, Helena, y Torrents, Dani (2018): “La evolución del acceso a la universidad en Cataluña: ¿Cómo la explicamos?" Revista Española de Sociología, 27 (1).

\section{Nota biográfica}

Manuel Tomás Valdés Fernández es licenciado en Ciencias Políticas y Sociología por la Universidad Carlos III de Madrid. Máster en Metodología de la Investigación en Ciencias Sociales por la Universidad Complutense de Madrid. Actualmente es doctorando en Sociología en la Universidad Complutense de Madrid, realizando su investigación doctoral en el marco del contrato para la Formación del Profesorado Universitario con referencia FPU16/02905. Líneas de investigación: Sociología de la Educación, estratificación y movilidad social. 